DOI: http://dx.doi.org/10.12957/demetra.2014.10659

\title{
Avaliação da prevalência de fatores de risco para o desenvolvimento de diabetes mellitus tipo 2 em pacientes da Clínica Unesc Saúde
}

\section{Evaluation of the prevalence of risk factors for development of type 2 diabetes mellitus in patients of the Unesc Clinic}

\author{
Arelli Bruno ${ }^{\prime}$ \\ Luciene Rabelo Pereira' \\ Herivelto dos Santos Almeida² \\ I Programa de Pesquisa e Pós-graduação, \\ Departamento de Nutrição. Clínica UNESC Saúde, \\ Unidade Honório Fraga. Centro Universitário do \\ Espírito Santo - UNESC. Colatina-ES, Brasil. \\ ${ }^{2}$ Departamento de Engenharia. Centro \\ Universitário do Espírito Santo - UNESC. \\ Colatina-ES, Brasil. \\ Correspondência /Correspondence \\ Arelli Bruno \\ E-mail: arellybruno@hotmail.com
}

\section{Resumo}

O diabetes mellitus tipo 2 é um problema de saúde pública com crescente número de pessoas afetadas cada vez mais jovens. Este estudo tem como objetivo conhecer a prevalência dos fatores de risco para esta patologia em pacientes que buscam atendimento médico ou nutricional na Clínica UNESC Saúde, no município de Colatina-ES. Foi conduzido estudo quantitativo transversal aleatório por meio da aplicação do questionário Finnish Diabetes Risk Score, que pontua os fatores de risco e os classifica em escore de risco para desenvolver o diabetes mellitus tipo $2 \mathrm{em} \mathrm{dez} \mathrm{anos.}$ A amostra foi composta por 100 pacientes, sendo $61,0 \%$ do sexo feminino e $39,0 \%$ do sexo masculino, com idade média de 38 anos e índice de massa corporal médio de $26,74 \mathrm{~kg} / \mathrm{m}^{2}$. Dentre os pacientes, $35,0 \%$ tinham idade $\geq 45$ anos; $62,0 \%$ estavam acima do peso; $60,0 \%$ possuem valor de circunferência de cintura aumentado; $89,0 \%$ não praticam ao menos 30 minutos de atividade física por dia; $15,0 \%$ não consomem diariamente vegetais, frutas, legumes ou grãos; $35,0 \%$ consomem frituras, salgados ou carnes gordas todos os dias; $6,0 \%$ são tabagistas, $21,0 \%$ relataram história de alteração glicêmica, diabetes gestacional ou macrossomia; 30,0\% fazem uso de anti-hipertensivos; 25,0\% referiam-se a familiares de $1^{\circ}$ grau com diabetes mellitus e $31,0 \%$ a familiares de $2^{\circ}$ grau. A elevada prevalência dos fatores de risco, sobretudo os de natureza modificável, ou seja, que são passíveis de intervenções no estilo de vida, confirma um importante campo de atuação para o nutricionista: a educação alimentar na prevenção de diversas patologias.

Palavras-chaves: Diabetes Mellitus. Saúde Pública. Fatores de Risco. Prevenção. Prevalência. 


\section{Abstract}

Type 2 diabetes mellitus is a public health problem with increasing number of young people affected. This study aims to determine the prevalence of risk factors for this condition in patients seeking medical or nutritional care in UNESC Clinic in the city of ColatinaES, Brazil. A random cross-sectional quantitative study applying the Finnish Diabetes Risk Score questionnaire, which evaluates the risk factors and classifies them into a risk score for developing diabetes mellitus type 2 at ten years was conducted. The sample consisted of 100 patients, $61.0 \%$ female and $39.0 \%$ male, mean age 38 years and mean body mass index of $26.74 \mathrm{~kg} / \mathrm{m}^{2}$. Among patients, $35.0 \%$ were aged $\geq 45$ years; $62.0 \%$ were overweight; $60.0 \%$ have increased value of waist circumference; $89.0 \%$ do not practice at least 30 minutes of physical activity per day; $15.0 \%$ do not consume vegetables, fruits, vegetables or grains daily; $35.0 \%$ consume fried foods, salty or fatty meats every day; $6.0 \%$ were smokers; $21.0 \%$ reported a history of glycemic change, gestational diabetes or macrosomia; $30.0 \%$ use anti-hypertensive drugs; $25.0 \%$ were related to $1^{\text {st }}$ degree relatives with diabetes mellitus, and $31.0 \%$ the family of $2^{\text {nd }}$ grade. The high prevalence of risk factors, especially of modifiable nature, ie, which are amenable to interventions in lifestyle, congirm an important field for the nutritionist: food education in the prevention of various diseases.

Key words: Diabetes Mellitus. Public Health. Risk Factors. Prevention. Prevalence.

\section{Introdução}

O processo de transição alimentar e nutricional refletido pelo atual padrão de consumo é baseado na substituição da ingesta de alimentos in natura, ricos em fibras, vitaminas e minerais, por alimentos industrializados, com altas concentrações de gorduras saturadas e carboidratos simples. Este fato, o aumento da expectativa de vida da população, o crescente número de pessoas com sobrepeso e obesidade e o estilo de vida sedentário, vêm sendo citados como importantes fatores de risco para as doenças crônicas não transmissíveis (DCNT), especificamente para diabetes mellitus tipo $2 \cdot^{1-3}$ 
De acordo com a Sociedade Brasileira de Diabetes (SBD), a população mundial de portadores de diabetes com idades entre 20 e 79 anos é de 371 milhões de pessoas. ${ }^{4}$ A posição ocupada pelo Brasil é a quarta entre os países com maior prevalência de diabetes, sendo responsável por 13,4 milhões de pessoas portadoras de diabetes nessa faixa etária, o que corresponde a aproximadamente $6,5 \%$ da população brasileira. Segundo a Agência Nacional de Saúde Suplementar (ANS), ${ }^{5}$ estima-se que $50 \%$ da população com diabetes desconhecem possuir a patologia, por ser a mesma ser assintomática em grande parte dos casos, de modo que algumas permanecem não diagnosticadas até demonstrarem sinais de complicações. ${ }^{4,5}$

O diabetes mellitus tipo 2 (DM 2) é uma patologia crônica, com desordem metabólica de etiologia múltipla, apresentando distúrbios no metabolismo de carboidratos, proteínas e lipídeos causados pela resistência tecidual periférica ou ausência de resposta insulínica associada a uma deficiência relativa da insulina, os quais cooperam para o desenvolvimento da hiperglicemia. ${ }^{6}$

O mais comum quando se manifesta a hiperglicemia é ter os dois distúrbios, tanto na ação como na secreção de insulina; no entanto há o predomínio de um deles. Pacientes com DM 2 não necessitam de tratamento com insulina exógena para sobreviver, como os pacientes de diabetes mellitus tipo 1, mas para o controle metabólico adequado, ela pode ser necessária, por isso o DM 2 é também chamado de diabetes não insulinodependente. A dependência de insulina pode vir a ocorrer em casos muito avançados e descontrolados da doença, quando as intervenções no estilo de vida e a medicação não se mostram suficientes para o controle glicêmico. ${ }^{7}$

Estudar os fatores de risco para uma patologia significa estudar a probabilidade de ocorrer determinado evento. Epidemiologicamente, o termo é usado para predizer a probabilidade de indivíduos saudáveis, mas em exposição a determinados fatores, desenvolverem dada doença. Não significa que necessariamente esta vá ocorrer, e sim que a presença de tais fatores torna o indivíduo mais vulnerável e propício a manifestar a doença. Um fator de risco "x" pode ser o desencadeador de várias doenças, assim como vários fatores de risco podem cooperar para a gênese de uma doença em comum. . $^{5}$

Os fatores de risco de natureza não modificável ou irreversível se referem a particularidades do indivíduo, como idade, etnia, história familiar, diabetes gestacional prévio e história de macrossomia fetal. Mesmo sabendo que não se podem alterar esses fatores, existe a possibilidade de alterar o estilo de vida do indivíduo, evitando a manifestação do DM 2 precocemente. ${ }^{9,10}$ 
Os fatores de risco de natureza modificável podem receber intervenções preventivas com maior poder custo-efetivo. São os fatores suscetíveis ao tratamento, tais como hipertensão arterial e alterações glicêmicas, e a mudanças no estilo de vida, tais como obesidade, tabagismo, hábitos alimentares, sedentarismo, entre outros. Esses fatores de risco comuns e modificáveis estão na base das mais prevalentes doenças crônicas. ${ }^{5,10,11}$

\section{Metodologia}

Realizou-se pesquisa quantitativa transversal, com o método de abordagem aleatória, em pacientes que procuram atendimento nutricional ou de especialidade médicas na Clínica UNESC Saúde - Unidade Honório Fraga.

A Clínica UNESC Saúde oferece serviços gratuitos na área de saúde à população do município de Colatina e região, por meio dos profissionais e acadêmicos do Centro Universitário do Espírito Santo (UNESC), realizados conforme consulta previamente agendada por telefone. Os serviços de especialidades oferecidos pela clínica são: pediatria, ginecologia, neurologia, cardiologia, pequenas cirurgias, dermatologia, cirurgia vascular, urologia, enfermagem, fisioterapia e nutrição.

A amostra da pesquisa é constituída por 100 pacientes que buscaram esses serviços e que foram abordados de modo a respeitar os princípios éticos da não maleficência, beneficência, justiça e autonomia dos sujeitos, e cumprir as diretrizes e normas regulamentadoras de pesquisa envolvendo seres humanos. Todos os participantes, após serem devidamente orientados sobre o trabalho, assinaram o Termo de Consentimento Livre e Esclarecido. O projeto de pesquisa foi avaliado e aprovado pelo Comitê de Ética e Pesquisa em Seres Humanos do Centro Universitário do Espírito Santo, sob parecer número 409.343.

Para a fundamentação científica, a pesquisa de campo foi realizada por meio da aplicação do questionário FINDRISC (Finnish Diabetes Risk Score), adaptado à cultura e aos hábitos brasileiros, mas mantendo os cálculos originais, sendo o mesmo de acesso público. O questionário original foi desenvolvido em 2001 pela Sociedade de Diabetes Finlandesa e está disponível no site do International Diabetes Federation (IDF) para download em vários idiomas. O IDF descreve o questionário como instrumento eficaz que pode ser usado como base para o desenvolvimento de questionários nacionais, levando em consideração fatores locais. ${ }^{12-14}$ 
Cada questão do questionário é pontuada, e sua soma categoriza o paciente de acordo com o grau de risco em: baixo risco (menor que 7 pontos), estima-se que uma em cada cem pessoas desenvolverá a doença; pouco elevado (7 a 11 pontos), estima-se que uma em cada vinte e cinco pessoas desenvolverá a doença; moderado (12 a 14 pontos), estima-se que uma em cada seis pessoas desenvolverá a doença; alto (15 a 20 pontos), estima-se que uma em cada três pessoas desenvolverá a doença; e risco muito alto (maior que 20 pontos), estima-se que uma em cada duas desenvolverá DM 2 nos próximos dez anos. Além disso, é um questionário útil para a identificação de pacientes com diabetes não diagnosticada e pré-diabetes, com $85 \%$ de precisão. $^{12}$

Para avaliação antropométrica, considerou-se o resultado da divisão da grandeza do peso em quilogramas (Kg) pela altura ao quadro em metros (m) para mensurar o índice de massa corporal (IMC). Para aferição do peso corporal, utilizou-se uma balança mecânica para adulto (Welmy®) com capacidade para $150 \mathrm{Kg}$ e divisões de 100 gramas, disponível no local da pesquisa, estando os voluntários trajando roupas leves e com os pés descalços.

A estatura foi aferida utilizando o estadiômetro correspondente à balança, com divisão de 0,5 cm, com os voluntários descalços, peso igualmente distribuído entre os pés, os braços estendidos ao longo do corpo, em posição ereta, com os calcanhares unidos e tocando a haste vertical do estadiômetro, posicionando a cabeça no plano de Frankfurt. A classificação do IMC utilizada pela pontuação do questionário FINDRISC como ponto de corte foi: IMC menor que $25,0 \mathrm{~kg} / \mathrm{m}^{2}$, IMC entre 25 a $30,0 \mathrm{~kg} / \mathrm{m}^{2}$ e IMC superior a $30 \mathrm{~kg} / \mathrm{m}^{2}$.

Para avaliar a circunferência da cintura, foi utilizada uma fita antropométrica inelástica (Cardiomed@) com precisão de $0,1 \mathrm{~cm}$. A circunferência da cintura foi medida no maior perímetro abdominal entre a última costela e a crista ilíaca, conforme recomendação da Organização Mundial da Saúde (OMS). A leitura foi feita no momento da expiração e realizada no milímetro mais próximo.

As demais questões do questionário foram aplicadas pelo pesquisador com respostas objetivas. Como critérios de exclusão da pesquisa, não participaram da mesma gestantes, pessoas com menos de 18 anos de idade e voluntários com diagnóstico de DM 2. A coleta de dados teve a duração de um mês. Não há conflito de interesse dos pesquisadores com o foco da pesquisa.

Posteriormente à coleta dos dados, foi realizada, com auxílio do aplicativo Microsoft Excel®, a estatística descritiva das variáveis quantitativas, sendo os resultados apresentados de forma discursiva durante a análise. Também foi utilizado o software Minitab $15{ }^{\circledR}$ para aplicação do 
Teste Qui-quadrado para a independência ou associação que, segundo Fonseca e Martins, tem o objetivo de estudar a associação ou dependência entre duas variáveis. Com o mesmo software, desenvolveram-se os cálculos para a tabulação cruzada dos dados. ${ }^{15}$

\section{Resultados}

Dos participantes, o percentual de 61,0\% ( $\mathrm{n}=61)$ corresponde ao sexo feminino e 39,0\% ( $\mathrm{n}$

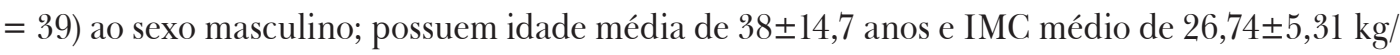
$\mathrm{m}^{2}$. A pontuação média dos pacientes da Clínica UNESC Saúde resultou em 10,52 $\pm 5,29$ pontos, o que equivale ao risco pouco elevado, com valor mínimo de 0 pontos e máximo de 26 pontos, moda e mediana igual a 9 .

Considerando a pontuação do questionário FINDRISC, 26,0\% dos participantes apresentam risco baixo; $35,0 \%$, risco pouco elevado; $12,0 \%$, risco moderado; $18,0 \%$, risco alto; e $9,0 \%$, risco muito alto de desenvolverem o DM 2 nos próximos dez anos.

Considerando os fatores de risco propostos pelo questionário, tem-se: 35,0\% dos pacientes com idade $\geq 45$ anos; $62,0 \%$ acima do peso; $60,0 \%$ com valor de circunferência de cintura aumentado; 89,0\% não praticam ao menos 30 minutos de atividade física diário; 15,0\% não consomem diariamente vegetais, frutas, legumes ou grãos; $35,0 \%$ consomem frituras, salgados ou carnes gordas todos os dias; 6,0\% são tabagistas; 21,0\% têm história de alteração glicêmica, diabetes gestacional ou macrossomia; $30,0 \%$ fazem uso de anti-hipertensivos; $25,0 \%$ referem-se a familiares de $1^{\circ}$ grau e $31,0 \%$ a familiares de $2^{\circ}$ grau com DM 2. Os dados podem ser visualizados no quadro 1. 
Quadro 1. Resultados da aplicação do Questionário FRINDRISC. Colatina-ES, 2012.

\begin{tabular}{|c|c|c|c|c|c|c|c|c|c|c|c|}
\hline & & \multicolumn{2}{|c|}{$\begin{array}{l}\text { Risco } \\
\text { baixo }\end{array}$} & \multicolumn{2}{|c|}{$\begin{array}{c}\text { Risco } \\
\text { pouco } \\
\text { elevado }\end{array}$} & \multicolumn{2}{|c|}{$\begin{array}{c}\text { Risco } \\
\text { moderado }\end{array}$} & \multicolumn{2}{|c|}{ Risco alto } & \multicolumn{2}{|c|}{$\begin{array}{c}\text { Risco } \\
\text { muito } \\
\text { alto }\end{array}$} \\
\hline \multicolumn{2}{|c|}{ Fator de Risco } & $\mathrm{n}$ & $\%$ & $\mathrm{n}$ & $\%$ & $\mathrm{n}$ & $\%$ & $\mathrm{n}$ & $\%$ & $\mathrm{n}$ & $\%$ \\
\hline \multicolumn{2}{|c|}{ GERAL } & 26 & 26,0 & 35 & 35,0 & 12 & 12,0 & 18 & 18,0 & 9 & 9,0 \\
\hline \multirow{7}{*}{ 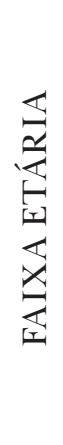 } & 18 a 24 anos & 12 & 46,1 & 4 & 11,4 & 2 & 16,7 & 2 & 11,1 & - & - \\
\hline & 25 a 32 anos & 4 & 15,4 & 11 & 31,4 & 3 & 25,0 & 3 & 16,7 & - & - \\
\hline & 35 a 44 anos & 6 & 23,1 & 13 & 37,1 & 2 & 16,7 & 3 & 16,7 & - & - \\
\hline & 45 a 54 anos & 4 & 15,4 & 5 & 14,3 & 2 & 16,7 & 5 & 27,8 & 3 & 33,3 \\
\hline & 55 a 64 anos & - & - & 1 & 2,9 & 2 & 16,7 & 3 & 16,7 & 4 & 44,4 \\
\hline & $>65$ anos & - & - & 1 & 2,9 & 1 & 8,3 & 2 & 11,1 & 2 & 22,2 \\
\hline & Total & 26 & 100,0 & 35 & 100,0 & 12 & 100,0 & 18 & 100,0 & 9 & 100,0 \\
\hline \multirow{3}{*}{$\begin{array}{l}0 \\
x \\
n \\
n\end{array}$} & Feminino & 14 & 53,9 & 13 & 37,1 & 3 & 25,0 & 6 & 33,3 & 3 & 33,3 \\
\hline & Masculino & 12 & 46,2 & 22 & 62,9 & 9 & 75,0 & 12 & 66,7 & 6 & 66,7 \\
\hline & Total & 26 & 100,0 & 35 & 100,0 & 12 & 100,0 & 18 & 100,0 & 9 & 100,0 \\
\hline & & & & & & & & & & & \\
\hline \multirow{5}{*}{$\underset{\Xi}{\Xi}$} & Baixo Peso & 1 & 3,9 & 1 & 2,9 & - & - & - & - & - & - \\
\hline & Eutrofia & 18 & 69,2 & 9 & 25,7 & 5 & 41,7 & 3 & 16,7 & 1 & 11,1 \\
\hline & Sobrepeso & 7 & 26,9 & 19 & 54,3 & 4 & 33,3 & 7 & 38,9 & 2 & 22,2 \\
\hline & Obesidade & - & - & 6 & 17,2 & 3 & 25,0 & 8 & 44,5 & 6 & 66,6 \\
\hline & Total & 26 & 100,0 & 35 & 100,0 & 12 & 100,0 & 18 & 100,0 & 9 & 100,0 \\
\hline
\end{tabular}




\begin{tabular}{|c|c|c|c|c|c|c|c|c|c|c|c|c|}
\hline \multirow{7}{*}{ 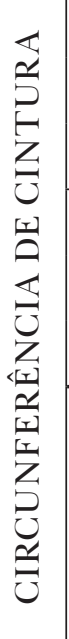 } & \multirow{3}{*}{ 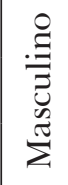 } & $<94 \mathrm{~cm}$ & 14 & 53,9 & 7 & 20,0 & 1 & 8,3 & 2 & 11,1 & - & - \\
\hline & & 94 a $102 \mathrm{~cm}$ & - & - & 2 & 5,7 & - & - & 1 & 5,6 & 1 & 11,1 \\
\hline & & $>102 \mathrm{~cm}$ & - & - & 3 & 8,6 & 1 & 8,3 & 3 & 16,7 & 2 & 22,2 \\
\hline & \multirow{3}{*}{ 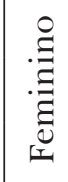 } & $<80 \mathrm{~cm}$ & 8 & 30,8 & 4 & 11,4 & 4 & 33,3 & - & - & - & - \\
\hline & & 80 a $88 \mathrm{~cm}$ & 4 & 15,4 & 6 & 17,1 & 2 & 16,7 & 3 & 16,7 & - & - \\
\hline & & $>88 \mathrm{~cm}$ & - & - & 13 & 37,1 & 4 & 33,3 & 9 & 50,0 & 6 & 66,7 \\
\hline & \multicolumn{2}{|r|}{ Total } & 26 & 100,0 & 35 & 100,0 & 12 & 100,0 & 18 & 100,0 & 9 & 100,0 \\
\hline & & & & & & & & & & & & \\
\hline \multirow{3}{*}{ 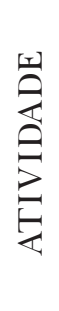 } & \multirow{3}{*}{ 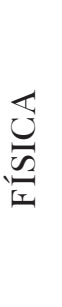 } & $\begin{array}{c}\text { Mais de } 4 \mathrm{~h} / \\
\text { semana }\end{array}$ & 7 & 26,9 & 2 & 5,7 & 1 & 8,3 & 1 & 5,6 & - & - \\
\hline & & $\begin{array}{c}\text { Menos de } 4 \\
\text { h/semana }\end{array}$ & 19 & 73,1 & 33 & 94,3 & 11 & 91,7 & 17 & 94,4 & 9 & 100,0 \\
\hline & & Total & 26 & 100,0 & 35 & 100,0 & 12 & 100,0 & 18 & 100,0 & 9 & 100,0 \\
\hline \multirow{3}{*}{ 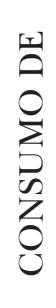 } & \multirow{3}{*}{ 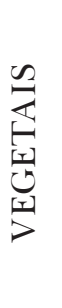 } & Dí́rio & 94 & 099 & 96 & 742 & 10 & 823 & 16 & 888 & 0 & 1000 \\
\hline & & Não diário & 2 & 7,7 & 9 & 25,7 & 2 & 16,7 & 2 & 11,2 & 0 & 0,0 \\
\hline & & Total & 26 & 100,0 & 35 & 100,0 & 12 & 100,0 & 18 & 100,0 & 9 & 100,0 \\
\hline \multirow{3}{*}{ 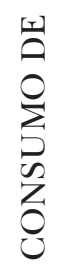 } & \multirow{3}{*}{ 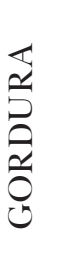 } & Diário & 8 & 30,7 & 15 & 42,8 & 6 & 50,0 & 5 & 27,8 & 1 & 11,1 \\
\hline & & Não diário & 18 & 69,3 & 20 & 57,2 & 6 & 50,0 & 13 & 72,2 & 8 & 88,9 \\
\hline & & Total & 26 & 100,0 & 35 & 100,0 & 12 & 100,0 & 18 & 100,0 & 9 & 100,0 \\
\hline
\end{tabular}




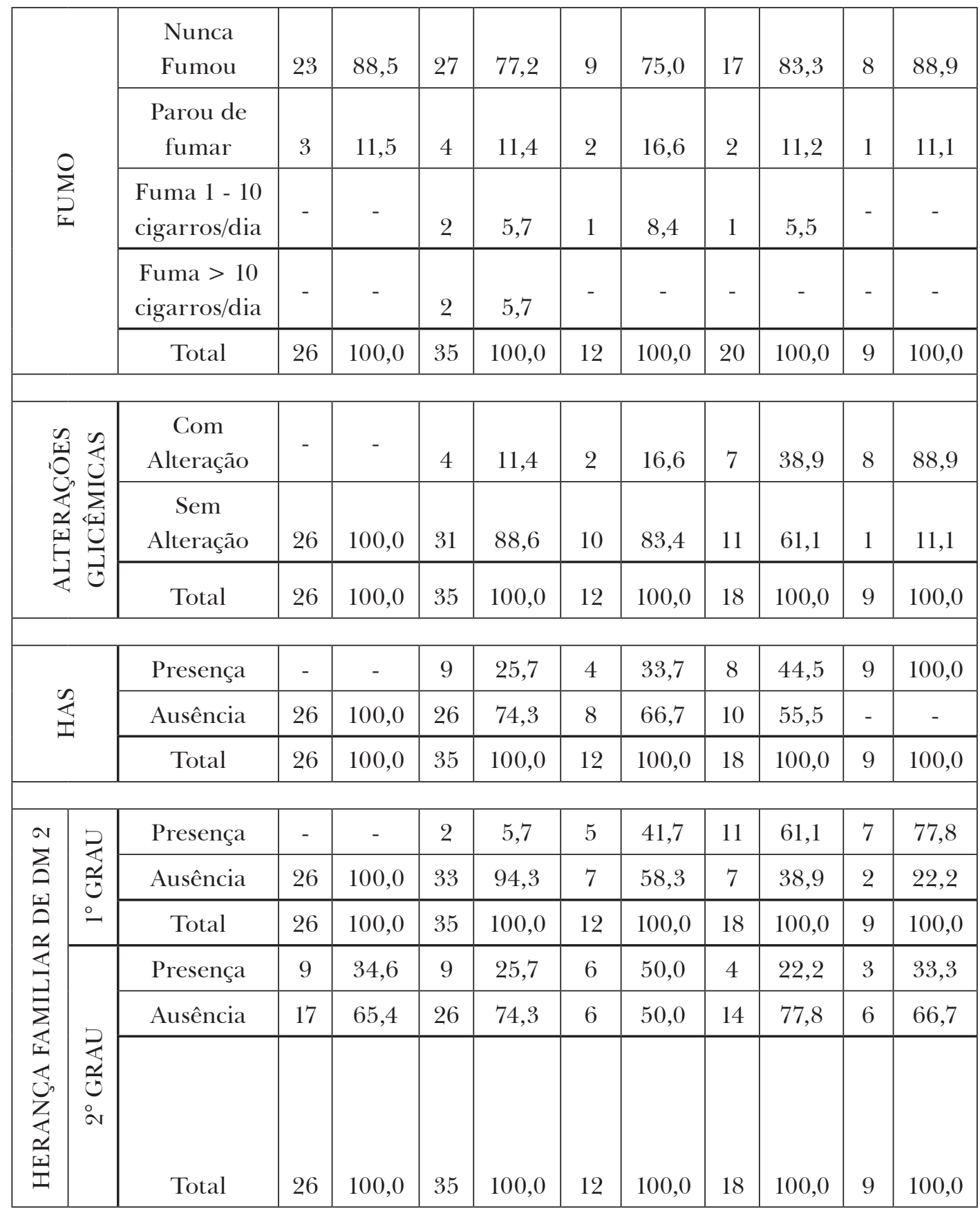




\section{Análise e discussão dos resultados}

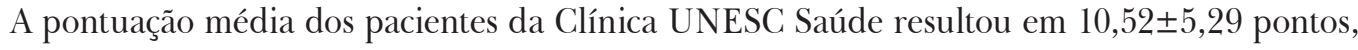
o que equivale ao risco pouco elevado. Valores médios similares foram encontrados por Winkler et al. ${ }^{16}$ em pesquisa realizada na Hungria, com pontuação média de 10,45 \pm 5,09 (entre 0 e 26 pontos), com percentual semelhante de participantes do sexo feminino $(60,1 \%)$ e masculino $(40,9 \%)$. Por sua vez, Lindström et al. encontraram FINDRISC médio de 13 (intervalo de 1 a 24 pontos) em uma população finlandesa. ${ }^{16,17}$

Considerando a pontuação do questionário FINDRISC com escore maior ou igual a 12 como risco de maior gravidade, e menor que 12 como menor gravidade, tem-se que 39,0\% (n = 39) da população estudada possuem maior risco de desenvolver a patologia nos próximos dez anos, distribuídos entre risco moderado, alto e muito alto, como visualizado no quadro 1. Esses achados são próximos aos encontrados por Winkler et al., segundo os quais 41,0\% dos pesquisados apresentavam risco aumentado. ${ }^{16}$

Quando verificada a gravidade de risco entre o sexo feminino e masculino, observa-se que apenas no grau de risco baixo o quantitativo de homens é maior que o de mulheres; em todos os demais estratos de risco, a proporção de mulheres é aproximadamente o dobro em relação aos homens. Na pesquisa VIGITEL 2011, foi revelado ser de 5,6\% a frequência de diagnóstico prévio de diabetes nas capitais brasileiras, sendo 6,0\% entre as mulheres e 5,2\% entre os homens. Diversos estudos apontam não haver diferença significativa de prevalência de DM em relação a sexo feminino ou masculino, e atribuem essas diferenças a maior procura e demanda dos serviços de saúde pelas mulheres, o que possibilita maior frequência de diagnóstico e acompanhamento

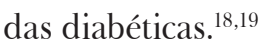

No estrato de risco baixo, a faixa etária predominante é dos 18 aos 44 anos de idade, com $84,7 \%$ dos pacientes desse grau de risco. Em contrapartida, a partir dos 45 anos, os estratos de riscos com maior quantitativo são risco alto $(55,6 \%)$ e risco muito alto $(100,0 \%)$. Ou seja, na faixa etária de 18 a 24 anos, à medida que aumenta o risco de desenvolver DM 2, decresce o quantitativo de pacientes nesse estrato; e a partir dos 55 anos de idade, este valor é crescente, com o aumento do grau de risco. Para tanto, considera o risco baixo como o estrato de menor gravidade de vir a manifestar a patologia, e o risco muito alto como a maior possibilidade.

Dados da VIGITEL 2011 revelam que o diagnóstico de DM 2 é mais comum a partir dos 35 anos para as mulheres e após os 45 anos para homens, de modo que mais de um quinto dos idosos referem ser diagnosticados como diabéticos. Estudo multicêntrico realizado em $1998 \mathrm{em}$ 
nove capitais brasileiras, para verificar a prevalência de DM, denominado Censo Brasileiro de Diabetes, demonstrou que na proporção em que aumenta a idade, eleva-se também a prevalência de DM, alcançando 17,4\% na faixa entre 60 a 69 anos. Em contrapartida, entre 30 a 59 anos, esse valor foi de apenas $2,7 \%{ }^{7,18}$

Outro trabalho realizado em Ribeirão Preto, utilizando a mesma metodologia do Censo Brasileiro de Diabetes, revelou entre a faixa etária de 30 a 69 anos uma prevalência de 12,1\% de diabetes e de $7,7 \%$ para tolerância diminuída a glicose. ${ }^{5}$

A ABESO utiliza pontos de cortes para classificação de IMC que fazem uma associação entre este e a doença crônica ou mortalidade. Neste sentido, na população estudada, apenas 36\% (n = 36) encontram-se eutróficos, ou seja, com o IMC entre 18,5 e $24,9 \mathrm{~kg} / \mathrm{m}^{2}$, de modo que $39 \%$ (n = 39) apresentam acúmulo excessivo de peso e $23 \%$ (n = 23) são classificados como obesos. Fator notável é que, à medida que se eleva o produto do peso em relação à altura, aumenta o número absoluto de pacientes nos estratos de maior risco. ${ }^{20}$

Como recomendado pela WHO, o IMC médio ideal para a população adulta se encontra no intervalo de 21 a $23 \mathrm{~kg} / \mathrm{m}^{2}$, uma vez que, conforme se afasta de $24,9 \mathrm{~kg} / \mathrm{m}^{2}$ o limite máximo para a eutrofia, mais susceptível se torna a desenvolver comorbidades correlacionadas ao acúmulo excessivo de peso, dentre elas o DM 2, visto que o peso, além de ser fator de risco fortemente associado a esta patologia, colabora para potencializar outros fatores. ${ }^{21}$

Apesar de o IMC ser bom indicador, o mesmo não está inteiramente correlacionado com a gordura corporal, tendo como uma das limitações o fato de não refletir, obrigatoriamente, a distribuição corporal de gordura, uma vez que a obesidade localizada na região intra-abdominal, ou seja, a gordura visceral, é potencial fator de risco que independe do total de gordura corporal. Deste modo, foi proposta a utilização do IMC em combinação com a circunferência abdominal, pois esta reflete o conteúdo de gordura visceral e a associa ao tecido adiposo total. Assim, considera-se como ponto de corte para avaliar obesidade e risco de DM 2: a circunferência da cintura entre 80 a $88 \mathrm{~cm}$ para mulheres, e 94 a $102 \mathrm{~cm}$ para homens, como risco aumentado de complicações metabólicas, e maior ou igual a 88 e $102 \mathrm{~cm}$ para o sexo feminino e masculino, respectivamente, como risco de complicações metabólicas aumentado substancialmente. ${ }^{20}$

Os pacientes em estudos têm ilustrado este relato, permitindo uma relação entre aumento da circunferência da cintura e consequente risco mais elevado para DM 2. Um percentual de 61,5\% ( $\mathrm{n}=24)$ dos homens apresentou CA inferior a $94 \mathrm{~cm}$, sendo que 26,2\% ( $\mathrm{n}=16)$ das mulheres apresentaram CA menor que $80 \mathrm{~cm}$. Maior percentual de homens $(35,9 \%)$ apresentou risco baixo 
com circunferência abdominal menor que $94 \mathrm{~cm}$ - o maior valor agrupado para o sexo feminino $(21,3 \%)$ refere-se à circunferência maior que $88 \mathrm{~cm}$ na classificação de risco pouco elevado, seguido por $14,7 \%$ ( $\mathrm{n}=9$ ) com o mesmo ponto de corte para CA com risco alto de vir a tornar-se diabético.

Marinho, em pesquisa no município de Itapipoca-CE entre os usuários da Estratégia da Saúde na Família com o mesmo instrumento de coleta de dados, encontrou 41,1\% da amostra com sobrepeso; 18,6\% obesos; 16,0\% com CA normal (menor que $80 \mathrm{~cm}$ para mulheres e menor que $94 \mathrm{~cm}$ para homens) e 84,0\% com CA elevado, sendo que 24,8\% apresentavam risco aumentado e $59,2 \%$, risco substancialmente aumentado para DM 2. Na atual pesquisa, 19,0\% apresentam risco elevado e 41,0\%, risco substancialmente elevado para desencadear o DM 2 em relação a CA. ${ }^{22}$

Segundo Gomes et al., o excesso de peso, juntamente com o acúmulo de adipócitos no região mesentérica, caracteriza obesidade do tipo androgênica ou visceral, a qual está relacionada com maior mortalidade em relação à obesidade periférica. Essa diferença se deve ao fato de o tecido adiposo visceral ser metabolicamente mais ativo, comparado ao tecido adiposo subcutâneo, levando, por exemplo, a uma produção elevada de glicose e, por consequência, DM 2 e hiperinsulinismo. ${ }^{23}$

A prática de exercício físico por quatro horas semanais ou mais dos pacientes atendidos pela Clínica UNESC Saúde revela que apenas $11 \%$ se exercitam diariamente, valor inferior ao encontrado na pesquisa de Marinho, de $16,7 \%$ da amostra. O maior quantitativo de praticantes de atividade física semanal $(n=7)$ encontra-se no estrato de risco baixo para desenvolvimento de DM 2, aumentando o número de sedentários conforme aumenta o grau de risco. O percentual de sedentarismo é superior ao estipulado por Webber et al., que afirmam ser comum o sedentarismo entre 30 a 60\% da população, os quais não cumprem a recomendação de atividade física que, segundo a Sociedade Brasileira de Endocrinologia e Metabolia et al., seria a realização de ao menos 150 minutos semanais de exercícios aeróbicos, com o objetivo de prevenir o desenvolvimento de doenças crônicas. ${ }^{22,24,25}$

A atividade física insuficiente é o quarto fator de risco principal para a mortalidade, de modo que pessoas insuficientemente ativas fisicamente apresentam risco de mortalidade de $20 \%$ a $30 \%$ maior por qualquer causa, quando comparadas àquelas que desenvolvem ao menos 30 minutos de atividade física de intensidade moderada na maior parte dos dias da semana. A participação em 150 minutos semanais de atividade moderada reduz o risco de diabetes em 27\%. Além do mais, a atividade física regular reduz o risco de acidente vascular cerebral, hipertensão e depressão, e é fator importante no gasto de energia, sendo fundamental para o balanço energético e controle de peso. ${ }^{21}$

Levando em consideração a alta prevalência de sedentarismo juntamente com o significativo risco de doenças crônico-degenerativas a que está exposta a população, o aumento da prática de exercícios físicos reflete a qualidade de saúde da coletividade, minimizando gastos com tratamentos. ${ }^{5}$ 
Quando questionados sobre o consumo diário de vegetais, frutas, legumes ou grãos, 85,0\% referiram o consumo de pelo menos um dos três grupos, de modo que o maior percentual $(9,0 \%)$ dos que relataram não consumir esses alimentos encontra-se no estrato de risco pouco elevado. Quanto ao consumo diário de frituras, salgados ou carnes gordas, incluindo pele de frango, $65,0 \%$ relataram não consumir nenhuma destas preparações diariamente, sendo que os que consomem diariamente o maior número $(\mathrm{n}=15)$ encontram-se no estrato de risco pouco elevado.

Contrariando os achados sobre o consumo alimentar nesta população, Rtveladze et al. revelam que a dieta do brasileiro sempre foi constituída por baixo consumo de verduras e alto de sal e açúcar, mas que num período de 16 anos, o consumo de vegetais e frutas apresentou queda de $20 \%$, aumentando nesse mesmo período em $21 \%$ o consumo de pães e biscoitos, fonte de calorias de custo menor no país, o que contribui para a elevação das taxas de obesidade. ${ }^{3}$

A Pesquisa de Orçamentos Familiares (POF) 2008-2009 afirma que 90\% da população brasileira têm ingesta diária de verduras, legumes e frutas abaixo dos níveis recomendados pelo MS (400g), sendo a base da dieta a combinação de arroz e feijão, juntamente com alimentos ricos em calorias e com poucos nutrientes. Assim, as principais inadequações de consumo alimentar pela população referem-se ao excesso de gorduras saturadas $(82,0 \%)$ e de açúcar (61,0\%), bem como à escassez de fibras $(68 \%)$ na dieta dos brasileiros. ${ }^{26}$

A pesquisa VIGITEL 2011 revela, ainda, que o consumo regular de frutas e hortaliças é maior entre as faixas etárias de 18 a 24 anos e após os 65 anos. Assim, a faixa etária fornecedora de mão de obra está exposta ao baixo consumo de vegetais. Também no grupo com idade entre 18 e 44 anos para ambos os sexos, observou-se maior consumo de carnes com excesso de gordura, consideradas como carnes vermelhas gordurosas ou frango com pele, sem remoção da gordura visível destes alimentos. O consumo de leite integral tende a ser menor com o aumento da idade, com frequências pequenas no estrato de maior escolaridade e máxima no estrato de escolaridade intermediária. O consumo de refrigerantes e sucos artificiais, por sua vez, foi referido em 80\% dos pesquisados em pelo menos um dia da semana e 29,8\% pelo menos cinco vezes por semana, tendendo a diminuir com a idade e o aumento do nível de escolaridade. ${ }^{18}$

O fato de a maior parte dos brasileiros consumir cotidianamente arroz e feijão pode ter influenciado na resposta dos pesquisados quando interrogados sobre o consumo diário de vegetais, frutas, legumes ou grãos, uma vez que a maioria dos pacientes que respondeu a essa questão relatou consumir tais tipos de alimentos de um grupo ou de outro, e não o consumo dos três grupos, como direcionado pela pirâmide alimentar. Vale ressaltar ainda que a pergunta pretendia apenas conhecer se existia ou não o consumo diário destes, não dando mérito à quantidade recomenda pela OMS como meio de prevenção de males crônicos. 
Segundo afirmação de Carolino et al., ${ }^{1}$ dentre os fatores de risco que são passíveis de modificação, o consumo alimentar habitual é o principal, assim como o controle de peso e o aumento da prática de exercício físico, que diminuem a resistência à insulina e, por consequência, tornam o indivíduo menos propenso a desenvolver o DM 2, até mesmo aqueles com história familiar da patologia.

Em relação ao tabagismo, apenas 6,0\% relataram fazer uso de cigarro diariamente e $12 \%$ deixaram de fumar nos últimos anos, sendo que o maior quantitativo de pessoas que pararam de fumar $(n=4)$ e o das que ainda fumam $(n=4)$ encontram-se no estrato de risco pouco elevado.

Nos países desenvolvidos, os fumantes somam 42,0\% dos homens e 24,0\% das mulheres; nos países em desenvolvimento, a participação do sexo feminino é em menor escala, perfazendo total de 7,0\% de mulheres e 48,0\% de homens fumantes. Recente pesquisa realizada no Brasil, pelo Instituto Nacional de Câncer, revelou que 22,7\% dos homens e 16,0\% das mulheres são fumantes - isso se refere a um total de $18,8 \%$ de brasileiros fumantes.

Os resultados encontrados em relação à prevalência de fumantes são inferiores aos encontrados pelo VIGITEL 2011, que revelou frequência de fumantes de 10,3\%, com tendência a ser maior entre a faixa etária de 45 a 54 anos e entre homens e mulheres com até oito anos de escolaridade. A pesquisa também revelou que a frequência de ex-fumantes foi de 22,4\%, maior entre os homens do que entre as mulheres. ${ }^{18}$

Estudo prospectivo envolvendo 7.735 homens com faixa etária entre 40 a 59 anos, em 24 cidades britânicas, avaliou o impacto da interrupção do fumo quanto ao risco do desenvolvimento de DM 2 e observou que as vantagens só se tornavam evidentes cinco anos após essa interrupção, uma vez que inicialmente apresentam maior ganho de peso e assim desenvolvem risco maior para o diabetes do que os que continuam fumando. O risco, no entanto, só foi revertido para igual risco dos que nunca fumaram 20 anos depois da suspensão do fumo. Embora não haja relação causal direta entre tabagismo e DM 2, diversos estudos demonstram que o cigarro eleva a concentração de gordura abdominal, diminui a sensibilidade insulínica e aumenta a concentração glicêmica, de modo que se atribui o risco à quantidade de cigarros e à duração do tabagismo. ${ }^{27,28}$

O questionário FRINDRISC considera como alterações glicêmicas a tolerância à glicose diminuída e a glicemia de jejum alterada, incluindo ainda, para o sexo feminino, a presença de DM durante a gestação e/ou filhos com mais de 4,0 kg de peso ao nascer. Desse modo, 21,0\% dos participantes relataram história de alteração glicêmica, sendo prevalentes nos grupos de risco alto $(\mathrm{n}=7)$ e risco muito alto $(\mathrm{n}=9)$ para o desenvolvimento da patologia em questão.

Mariath et al., ${ }^{29} \mathrm{em}$ trabalho realizado com o objetivo de avaliar o estado nutricional e identificar os principais fatores de risco para DCNT em colaboradores da fábrica e do escritório de uma indústria localizada em Jaguará do Sul, no norte do estado de Santa Catarina, encontraram valor 
médio de glicemia capilar de 89,01 $\pm 16,30 \mathrm{mg} / \mathrm{dL}$, mas $4 \%$ da amostra apresentaram glicemia de jejum alterada.

Amorim et al. ${ }^{30}$ realizaram trabalho cujo objetivo foi determinar a frequência de macrossomia nos recém-nascidos vivos em um serviço obstétrico de referência em Campina Grande-PB e sua associação com fatores de risco maternos, do qual participaram 551 puérperas internadas. Encontraram frequência de 5,4\% para macrossomia, associada a fatores como sobrepeso e obesidade pré-gestacional, ganho ponderal excessivo, hipertensão e diabetes clínica ou gestacional.

Valores glicêmicos elevados para o normoglicêmico, mas inferiores aos requisitados para diagnóstico de diabetes, podem e devem ser utilizados para retardar ou prevenir a progressão para DM.,25

A condição de HAS e indivíduos normotensos em uso de medicação anti-hipertensiva está presente em 30,0\% ( $\mathrm{n}=30)$ dos pacientes e ausente nos indivíduos classificados como risco baixo; em contrapartida, está presente em todos os pesquisados $(n=9)$ do estrato de risco muito alto.

Como referenciado por Francisco et al., ${ }^{31}$ o quadro hipertensivo associa-se ao maior grau de resistência insulínica, de modo que os medicamentos anti-hipertensivos complicam esse processo, o que consequentemente torna o hipertenso três vezes mais susceptível ao desenvolvimento de DM. Essa associação preocupa ainda mais à medida que a hipertensão em indivíduos diabéticos eleva o risco de complicações cardiovasculares, tais como aterosclerose e acidente vascular cerebral.

Seguindo o critério de diagnóstico $\geq 140 / 90 \mathrm{mmHg}$ para hipertensão arterial sistêmica, sua prevalência varia de 22,3\% a 43,9\% na população adulta brasileira, dependendo da cidade em estudo. Alguns estudos demonstram taxas de prevalência de 20,0\% sem distinção de sexo, mas com clara tendência de elevar-se conforme a idade. Esses índices sofrem variação de acordo com a população estudada, mas são prevalentes em demais países da América Latina. ${ }^{5}$

Marinho, ${ }^{22}$ em seu trabalho, encontrou 12,9\% dos participantes em uso de anti-hipertensivos. Vilarinho et al., ${ }^{9} \mathrm{em}$ pesquisa com acadêmicos de Enfermagem para identificação de fatores de risco para DM 2, encontraram 57,5\% destes com referência de DM 2 em familiares e 75,0\% referiram casos de hipertensão. Não obstante, 45,0\% dos participantes ainda relataram casos simultâneos de diabetes mellitus e hipertensão.

No atual trabalho, quando interrogados sobre a presença de DM 2 em familiares de $1^{\circ}$ grau, definidos como pai, filhos e irmãos, 25,0\% $(\mathrm{n}=25)$ dos pacientes relataram possuir familiares com a patologia. Observa-se que em indivíduos com risco alto e muito alto, esse fator genético está presente em 61,11\% e 77,78\% dos pacientes, respectivamente. Por sua vez, 31,0\% dos pacientes relataram a presença da patologia nos familiares de $2^{\circ}$ grau, considerados como avós, tios e tias e primos em primeiro grau, sendo que, nos pacientes de risco moderado a muito alto, esta condição esteve presente em 33,33\% ( $\mathrm{n}=13)$ destes. 
Crispim et al., em trabalho realizado com a população do sul do Brasil, encontraram ao menos $76,6 \%$ dos pacientes diabéticos com relatos da presença desta patologia em pelo menos um familiar de primeiro grau, de modo que 9,9\% relataram apenas o pai diabético e 35,2\% apenas a mãe com DM 2, enquanto $10 \%$ apresentavam ambos os pais como portadores da doença. Padrão semelhante é observado ao se ter maior histórico familiar de diabetes em parentes maternos $(14,9 \%)$ do que em paternos $(6,1 \%)$, sugerindo transmissão materna do DM 2, o que também já foi acordado em estudos anteriormente citados. ${ }^{31}$

Guerra-Juárez, Gallegos e Cerda-Flores ${ }^{32}$ relataram que os riscos de familiares diretos de um adulto diabético tipo 2 desenvolverem a doença são mais elevados quando estes também apresentam sobrepesos ou obesidade Reafirmam, assim, a necessidade de cautela ao atribuir unicamente ao fator genético o aumento da prevalência e incidência do DM 2, mas que indivíduos geneticamente predispostos tornam-se mais vulneráveis a desenvolver a doença quando expostos a fatores ambientais.

No presente estudo, observou-se associação estatisticamente significativa entre as variáveis referentes a alterações glicêmicas, uso de anti-hipertensivos e DM 2 em familiares de $1^{\circ}$ grau e o grau de risco para o desenvolvimento desta patologia nos próximos dez anos. As variáveis "sexo", "consumo diário de vegetais, frutas, legumes ou grãos", "consumo diário de frituras, salgados e carnes gordas" e "DM 2 em familiares de $2^{\circ}$ grau" não mostraram dependência ou associação ao grau de risco, conforme a tabela 1. A mensuração das demais variáveis (faixa etária, IMC, CA e fumo) não foi possível devido ao tamanho da amostra, visto não ser aconselhável aplicar o teste qui-quadrado de associação em frequências menores do que $5 .^{15}$

Tabela 1. Associação estatística entre variáveis e risco de desenvolver DM 2. Colatina-ES, 2012.

\begin{tabular}{lc}
\hline \multicolumn{1}{c}{ Variáveis } & $\begin{array}{c}\text { Teste Qui-quadrado de } \\
\text { associação }\end{array}$ \\
\hline Sexo & $\mathrm{P}=0,432$ \\
Consumo diário de vegetais, frutas, legumes ou grãos & $\mathrm{P}=0,194$ \\
Consumo diário de frituras, salgados e carnes gordas & $\mathrm{P}=0,286$ \\
História de alteração glicêmica & $\mathrm{P}=0,000$ \\
H.A e uso de anti-hipertensivos & $\mathrm{P}=0,000$ \\
DM 2 em familiares de $1^{\circ}$ grau & $\mathrm{P}=0,000$ \\
DM 2 em familiares de $2^{\circ}$ grau & $\mathrm{P}=0,570$ \\
\hline
\end{tabular}


Estudo promovido pela Universidade Federal do Ceará, por um grupo de pesquisa em diabetes, em funcionários do Hospital Estadual de Messejana, demonstrou não haver associação estatística significante entre as variáveis "sexo" e "idade" com o grau de risco para diabetes. No entanto, confirmou a associação entre risco de diabetes e hipertensão arterial $(p=0,041)$, nível de glicemia $(\mathrm{p}=0,000)$ e hábito de fumar $(\mathrm{p}<0,05){ }^{33}$

\section{Conclusão}

A partir do que foi exposto e discutido ao longo deste trabalho, sobre a magnitude do DM 2, conclui-se que os fatores de risco para tal patologia estão cada vez mais prevalentes entre a população, principalmente quanto aos fatores de risco modificáveis - ou seja, os que são passíveis de intervenções e tratamento, mas que perfazem a base das doenças crônicas.

Como evidenciado pelo próprio nome, os fatores de risco não modificáveis são irreversíveis, mas pode e deve ser modificado o estilo de vida do paciente, de modo a evitar a manifestação precoce da patologia. Essas intervenções são referentes sobretudo a redução de peso, manutenção do peso perdido, restrição energética moderada, aumento da atividade física regular, estímulo à ingestão de fibras, ácidos graxos monoinsaturados e poli-insaturados, antioxidantes e minerais e restrição ao consumo de gorduras saturadas, gorduras trans, colesterol e sódio. Portanto, a educação alimentar é um dos pontos primordiais para a prevenção primária das DCNT e, particularmente, do DM 2, inclusive em pacientes em alto risco de desenvolvimento da patologia, nos quais pequenas modificações implicam prevenir, ou ao menos retardar, a progressão e o desenvolvimento da patologia.

Por fim, frente aos fatores de risco para o desenvolvimento do DM 2 e a importância da mudança de hábitos alimentares nesse contexto, este trabalho ainda reforça a importância da atuação do profissional de nutrição na atenção básica de saúde, seja no atendimento individualizado ou como integrante de uma equipe multidisciplinar.

\section{Referências}

1. Carolino IDR, Molena-Fernandes CA, Tasca RS, Marcon SS, Cuman RKN. Fatores de risco em pacientes com diabetes mellitus tipo 2. Rev Latino-am Enfermagem [Internet]. 2008; 16 (2). [Acesso em 8 ago. 2013]. Disponível em: http://www.scielo.br/pdf/rlae/v16n2/pt_11.pdf.

2. Carvalho GQ, Alfemas RCG. Índice glicêmico: uma abordagem crítica acerca de sua utilização na prevenção e no tratamento de fatores de fatores de risco cardiovasculares. Rev. Nutr. 2008; 21(5):577-87. 
3. Rtveladze K, Marsh T, Webber L, Kilpi F, Levy D, McPherson WK, et al. Health and economic burden of obesity in Brazil. PLoS One 2013; 8(7):1-7.

4. Sociedade Brasileira de Diabetes. São 13.4 milhões de pessoas portadoras de diabetes no Brasil [Internet]. 2013. [Acesso 15 jul. 2013]. Disponível em: http://www.diabetes.org.br/ultimas/sao-134-milhoes-de-pessoas-portadoras-de-diabetes-no-brasil

5. Brasil. Ministério da Saúde. Agência Nacional de Saúde Suplementar. Manual técnico para promoção da saúde e prevenção de riscos e doenças na saúde suplementar [Internet]. 4. ed. Rio de Janeiro: ANS; 2011. [acesso em 13 out. 2013]. Disponível em: http://bvsms.saude.gov.br/bvs/publicacoes/ manual_tecnico_promocao_saude_4ed.pdf

6. Mello VD, Laaksonen DE. Fibras na dieta: tendências atuais e benefícios à saúde na síndrome metabólica e no diabetes melito tipo 2. Arq. Bras. Endocrinol. Metabol. 2009; 53(5):509-18.

7. Sociedade Brasileira de Diabetes. Diretrizes Sociedade Brasileira de Diabetes, 2009 [Internet]. Disponível em: http://www.prograd.uff.br/farmacoclinica/sites/default/files/diretrizes09_final_0.pdf

8. Almeida VCF. Ocupação e fatores de risco para Diabetes Mellitus tipo 2: contribuição ao estudo do processo saúde-doença de trabalhadores de enfermagem [Tese] [Internet]. Fortaleza: Centro de Ciências da Saúde, Universidade Estadual do Ceará; 2007. [Acesso em 21 jun. 2013]. Disponível em: http://www.repositorio.ufc.br/ri/bitstream/riufc/2153/1/2007_tese_vcfalmeida.pdf

9. Vilarinho RMF, Lisboa MTL, Thiré PK, França PV. Prevalência de fatores de risco de natureza modificável para a ocorrência de diabetes mellitus tipo 2. Esc. Anna Nery Rev. Enferm. 2008; 12(3):452-56.

10. Alves A, Marques IR. Fatores relacionados ao risco de doença arterial coronariana entre estudantes de enfermagem. Rev. Bras. Enferm. 2009; 62(6):883-88.

11. Malta DC, Cezário AC, Moura L, Morais Neto OL, Silva Júnior JB. A construção da vigilância e prevenção das doenças crônicas não transmissíveis no contexto do Sistema Único de Saúde. Epidemiologia e Serviços de Saúde 2006; 15(3):47-65.

12. São Paulo. Prefeitura. Telessaúde. Núcleo São Paulo. Diabetes. Questionário: risco de ter diabetes [Internet]. Disponível em: http://www.prefeitura.sp.gov.br/cidade/secretarias/upload/saude/ arquivos/programas/Questionario_Risco_de_ter_Diabetes_Telessaude.pdf

13. International Diabetes Federation. Diabetes Questionnaire [internet]. Brussels: IDF; 2013 [Acesso 19 mar. 2013]. Disponível em: http://www.idf.org/diabetes-prevention/questionnaire

14. International Diabetes Federation. About diabetes [Internet]. Brussels: IDF; 2013. [acesso 03 mar. 2013]. Disponível em: http://www.idf.org/about-diabetes.

15. Fonseca JS, Martins GA. Curso de estatística. 6. ed. São Paulo: Atlas; 2010.

16. Winkler G, Hidvégi T, Vándorfi G, Balogh S, Jermendy G. Prevalence of undiagnosed abnormal glucose tolerance in adult patients cared for by general practitioners in Hungary. Results of a riskstratified screening based on FINDRISC questionnaire. Med. Sci. Monit. 2013; 19:67-72. 
17. Lindström J, Peltonen M, Eriksson JG, Aunola S, Hämäläinen H, Ilanne-Parikka P, et al. Determinants for the effectiveness of lifestyle intervention in the finnish diabetes prevention study. Diabetes Care 2008; 31(5):857-62.

18. Brasil. Ministério da Saúde. Vigitel Brasil 2011: Vigilância de fatores de risco e proteção para doenças crônicas por inquérito telefônico. Brasília: Ministério da Saúde; 2012. 131 p.

19. Fereirra CLR, Fereirra MG. Características epidemiológicas de pacientes diabéticos da rede pública de saúde: análise a partir do sistema HiperDia. Arq. Bras. Endocrinol. Metabol. 2009; 53(1):80-86.

20. Associação Brasileira para o Estudo da Obesidade e da Síndrome Metabólica. Diretrizes brasileiras de obesidade 2009/2010 [Internet]. 3 ed. Itapevi, SP: AC Farmacêutica; 2009. [acesso em 22 set. 2013]. Disponível em: http://www.abeso.org.br/pdf/diretrizes_brasileiras_obesidade_2009_2010_1.pdf

21. World Health Organization. Diabetes [Internet]. [Acesso em 24 jun. 2013]. Disponível em: http://www.who.int/mediacentre/factsheets/fs312/en/

22. Marinho NBP. Avaliação do risco para diabetes mellitus tipo 2 entre adultos de Itapipoca, CE [Dissertação]. Fortaleza: Universidade Federal do Ceará. Programa de Pós-Graduação em enfermagem; 2010.

23. Gomes F, Telo DF, Souza HP, Nicolau JC, Halpern A, Serrano Júnior CV, et al. Obesidade e doença arterial coronariana: papel da inflamação vascular. Arq. Bras. Cardiol. 2010; 94(2):273-279.

24. Webber L, Kilpi F, Marsh T, Rtveladze K, Brown M, McPherson k. High rates of obesity and noncommunicable diseases predicted across Latin America. Plos One 2012; 7(8):e39589.

25. Sociedade Brasileira de Endocrinologia e Metabologia. Projeto diretrizes. Diabetes Mellitus tipo 2: prevenção. São Paulo: AMB; 2011.

26. Instituto Brasileiro de Geografia e Estatística. POF 2008 - 2009: mais de 90\% da população comem poucas frutas, legumes e verduras [Internet] [Acesso em 05 out. 2013]. Disponível em: http://saladeimprensa.ibge.gov.br/noticias?idnoticia=1937\&t=pof-20082009-mais-90-populacaocomem-poucas-frutas-legumes-verduras\&view=noticia

27. Wannamethee SG, Shaper AG, Perry IJ. Smoking as a modifiable risk factor for type 2 diabetes in middle-aged men. Diabetes Care 2001; 24(9):1590-95.

28. Lyra R, Oliveira M, Lins D, Cavalcanti N. Prevenção do Diabetes Mellitus tipo 2. Arq. Bras. Endocrinol. Metab. 2006; 50(2):239-249.

29. Mariath AB, Grillo LP, Silva RO, Schimitz P, Campos IC, Medina JRP, et al. Obesidade e fatores de risco para o desenvolvimento de doenças crônicas não transmissíveis entre usuários de unidade de alimentação e nutrição. Cad. Saúde Pública 2007; 23(4):897-905.

30. Amorim MMR, Leite DFB, Gadelha TGN, Muniz AGV, Melo ASO, Rocha AM. de et al. Fatores de risco para macrossomia em recém-nascidos de uma maternidade-escola no Nordeste do Brasil. Rev. Bras. Ginecol. Obstet. 2009; 31(5):241-8. 
31. Francisco PMS, Belon AP, Barros MBA, Carandina L, Alves MCGP, Goldbaum M, et al. Diabetes auto-referido em idosos: prevalência e fatores associados e práticas de controle. Cad. Saúde Pública 2010; 26(1):175-84.

32. Guerra-Juárez R, Gallegos EC, Cerda-Flores RM. Mudança em os padrões de vida em descendentes de progenitores com diabetes mellitus tipo 2 do nordeste do México. Rev. Latino-Am Enfermagem 2007; 15 (5):909-913.

33. Coelho MM, Silva LF, Lino RV, Almeida PC, Ribeiro ME, Caracas M, et al. Diabetes mellitus tipo 2: riesgo en funcionários de un hospital público (datos oficiales). Enfermería Integral 2005; (70):29-35.

Recebido: 26/4/2014

Revisado: 02/6/2014

Aprovado: 04/8/2014 